\title{
Dor e analgesia em pacientes com síndrome da imunodeficiência adquirida*
}

\author{
Pain and analgesia in patients with acquired immunodeficiency syndrome
}

Roberta Meneses Oliveira ${ }^{1}$, Lucilane Maria Sales da Silva², Maria Lúcia Duarte Pereira ${ }^{3}$, Josenília Maria Alves Gomes ${ }^{4}$, Sarah Vieira Figueiredo ${ }^{5}$, Paulo César de Almeida ${ }^{6}$

* Recebido do Hospital São José de Doenças Infecciosas e Parasitárias. Fortaleza, CE.

\section{RESUMO}

JUSTIFICATIVA E OBJETIVOS: Em pacientes com síndrome da imunodeficiência adquirida (SIDA), o subdiagnóstico e o subtratamento da dor são alarmantes e poucos estudos analisam esse tema, bem como os registros de sua ocorrência. O objetivo deste estudo foi analisar registros sobre dor e analgesia em prontuários de pacientes com SIDA internados.

MÉTODO: Pesquisa documental, com análise de 63 prontuários, realizado em hospital de referência no tratamento da SIDA no Ceará, em 2010. Utilizou-se check-list para obtenção de dados e os resultados foram apresentados em tabelas com frequências relativo/absoluta.

1. Mestre em Cuidados Clínicos em Saúde. Doutoranda do Programa de Pós-Graduação Cuidados Clínicos em Enfermagem e Saúde (PPCCLIS), Universidade Estadual do Ceará (UECE). Fortaleza, CE, Brasil.

2. Doutora em Enfermagem. Coordenadora e Docente do Programa de Pós-Graduação Cuidados Clínicos em Enfermagem e Saúde (PPCCLIS), Universidade Estadual do Ceará (UECE). Fortaleza, CE, Brasil.

3. Doutora em Enfermagem. Docente do Curso de Graduação em Enfermagem e do Programa de Pós-Graduação Cuidados Clínicos em Enfermagem e Saúde (PPCCLIS), Universidade Estadual do Ceará (UECE). Coordenadora de Ensino e Pesquisa do Hospital São José de Doenças Infecciosas. Fortaleza, CE, Brasil.

4. Doutora em Clínica Cirúrgica. Professora Assistente do Curso de Medicina da Universidade de Fortaleza. Fortaleza, CE, Brasil. 5. Enfermeira. Membro do Grupo de Pesquisa Cuidados à Saúde da Criança e do Adolescente e Enfermagem, Universidade Estadual do Ceará (UECE). Fortaleza, CE, Brasil.

6. Doutor em Saúde Pública. Professor Adjunto da Universidade Estadual do Ceará (UECE). Fortaleza, CE, Brasil.

Endereço para correspondência:

Dra. Roberta Meneses Oliveira

Rua Lídia Brígido, 837 - Bairro Cidade dos Funcionários

60821-800 Fortaleza, CE.

Fone: (85) 8725-3983

E-mail: menesesroberta@yahoo.com.br
RESULTADOS: Encontrou-se registro de dor na maioria dos prontuários $(90,5 \%)$, especificando localização $(90,5 \%)$, fatores de melhora/piora $(55,6 \%)$, intensidade $(39,7 \%)$, frequência $(25,4 \%)$, entre outros aspectos. Foram responsáveis pelos registros médicos $(94,7 \%)$, enfermeiros $(87,8 \%)$ e fisioterapeutas $(12,2 \%)$. Quanto à localização, prevaleceu cefaleia $(50,9 \%)$, dor abdominal $(52,6 \%)$, torácica $(33,3 \%)$, membros inferiores $(24,6 \%)$ e lombalgia (29,8\%). Quanto à intensidade, dor forte (56\%), leve $(28 \%)$ e moderada (16\%). Quanto à duração, dor contínua $(62,5 \%)$ e intermitente $(37,5 \%)$. Nas prescrições farmacológicas, predominou anti-inflamatório não esteroide $(66,7 \%)$, seguido de analgésicos simples $(44,4 \%)$ e adjuvantes (41,3\%). Medidas não farmacológicas foram prescritas em apenas $11 \%$ dos prontuários.

CONCLUSÃO: É necessária a atenção dos profissionais para o registro de informações detalhadas das queixas álgicas dos pacientes com SIDA, com a adoção de instrumentos adequados para a avaliação e registro dos dados avaliados, para melhorar a assistência e o controle da dor que incide na maioria desses pacientes.

Descritores: Dor, Medição da dor, Registros como assunto, Síndrome da imunodeficiência adquirida.

\section{SUMMARY}

BACKGROUND AND OBJECTIVES: In acquired immunodeficiency syndrome (AIDS) patients, pain underdiagnostic and undertreatment are alarming and few studies have evaluated this subject, as well as the records of its incidence. This study aimed at analyzing records about pain and analgesia of hospitalized AIDS patients.

METHOD: Documental research with the analysis of 63 medical charts of an AIDS treatment reference hospital of Ceará, in 2010. Data were collected via checklist and results were presented in tables with relative/absolute frequencies. 
RESULTS: Most medical charts had pain records (90.5\%), specifying location (90.5\%), improvement/ worsening factors $(55.6 \%)$, intensity $(39.7 \%)$ and frequency (25.4\%), among other aspects. Responsible for medical charts were physicians $(94.7 \%)$, nurses $(87.8 \%)$ and physical therapists $(12.2 \%)$. Most frequent sites were headache $(50.9 \%)$, abdominal pain $(52.6 \%)$, chest $(33.3 \%)$, lower limbs $(24.6 \%)$ and low back pain $(29.8 \%)$. As to intensity, pain was severe (56\%), mild $(28 \%)$ and moderate $(16 \%)$. As to duration, pain was continuous $(62.5 \%)$ and intermittent (37.5\%). There has been predominance of non-steroid anti-inflammatory drugs (66.7\%), followed by common analgesics (44.4\%) and adjuvants (41.3\%). Non-pharmacological measures were prescribed in just $11 \%$ of medical charts.

CONCLUSION: Health professionals have to pay attention to the detailed recording of pain complaints of AIDS patients, with the adoption of adequate tools to evaluate and record evaluated data, to improve assistance and control pain affecting most of these patients.

Keywords: Acquired immunodeficiency syndrome, Pain, Pain measurement, Records as subject.

\section{INTRODUÇÃO}

Em todo o mundo, a dor é considerada uma das queixas mais comuns nos atendimentos de urgência e nos ambulatórios das diversas especialidades médicas e de outros profissionais de saúde.

No âmbito das doenças infecciosas, tem sido comumente relacionada aos pacientes portadores do vírus da imunodeficiência adquirida (HIV), com taxa aumentada de dor crônica, particularmente a neuropatia periférica. Além disso, esta dor é subtratada e mais difícil de manusear por uma série de razões, incluindo complexos regimes antirretrovirais, riscos mais elevados de efeitos colaterais, taxas mais altas de comorbidades psiquiátricas e abuso de substâncias ${ }^{1}$.

A explicação para a ocorrência de dor em portadores da síndrome da imunodeficiência adquirida (SIDA) encontra-se no fato de, à medida que o HIV altera o sistema imune de um indivíduo afetado, o número de infecções e malignidades aumenta e as sequelas geradas pela imunossupressão têm um sintoma comum frequente de dor. Deste modo, o predomínio da dor, nos indivíduos infectados pelo vírus, varia dependendo do estágio da doença, dos cuidados e da metodologia de tratamento ${ }^{2}$.

Diante do exposto, os profissionais que atuam na assistência aos pacientes portadores do HIV têm sido estimulados a avaliar a dor na rotina dos cuidados, com especial atenção às dores de caráter intermitente e crônico, que podem estar associadas à doença propriamente dita e/ou às infecções oportunistas ${ }^{3}$.

Para tanto, devem explorar suas queixas de dor, coletar dados sobre fatores agravantes, atenuantes e concomitantes; explorar indicativos de desconforto causado pela dor e utilizar-se de instrumentos que podem auxiliar na sua mensuração e avaliação, bem como na qualidade da analgesia ${ }^{4}$. Após colher tais dados, devem registrá-los prontamente em seus respectivos prontuários, a fim de que se tornem conhecidos e validados pela equipe interdisciplinar. Se existem sistematização, avaliação e registros precisos, a dor é mais bem identificada e tratada apropriadamente.

A queixa verbal é a forma mais clara e segura de avaliação da dor. Sendo assim, se o paciente tem dificuldade em expressá-la, a avaliação para o sujeito não se torna clara. A equipe de saúde deve, portanto, ser instruída a mensurar e registrar a dor no prontuário do paciente 5 . Desse modo, o prontuário do paciente é importante veículo de comunicação no meio hospitalar, reunindo documentos padronizados e organizados onde são registradas as ações prestadas durante sua internação. Neles é que se podem realizar anotações e fornecer informações a todos os componentes da equipe multiprofissional, dispostas em ordem cronológica ${ }^{6}$.

É imperativa a ampliação das discussões das interfaces da comunicação e da saúde, uma vez que a codificação do processo de comunicação interfere diretamente na recuperação do paciente, pois o serviço assistencial de saúde intenciona, através de atributos de eficiência e efetividade técnica, proporcionar um entendimento harmonioso entre ele e a equipe de enfermagem e de médicos e, consequentemente, melhor atendimento. Em outras palavras, a efetividade da comunicação entre a equipe multiprofissional de saúde e o paciente possibilita a sua melhora, devendo ser incentivada e aprimorada.

\section{MÉTODO}

Realizou-se estudo documental, prospectivo, de abordagem quantitativa, com base na análise dos prontuários de 63 pacientes atendidos durante os meses de junho a agosto de 2010 nas unidades de internação de um hospital de referência no tratamento de doenças infecciosas no estado do Ceará.

O quantitativo de prontuários (63) refere-se ao número de pacientes incluídos na amostra da pesquisa, calculado com base no cálculo de amostra com população finita. Segundo dados do hospital, no primeiro trimestre de 2010, foram internados 207 pacientes com diagnóstico de SIDA. Desse total, pelo cálculo de amostra com 
população finita, adotando-se prevalência de 50 e erro amostral de cinco, atingiu-se a amostra de 63 pacientes. Este quantitativo recebeu avaliação criteriosa dos registros em seus prontuários, desde o momento da internação até a data da coleta das informações.

Como critérios de inclusão, consideraram-se: prontuários dos pacientes internados há, pelo menos, um mês; com diagnóstico de SIDA há, pelo menos, seis meses; e que reunissem as características de preenchimento completo, legível e que contemplassem o preenchimento do checklist. Este instrumento de coleta de dados continha aspectos de relevância para a avaliação dos registros de dor em prontuários, divididos em duas categorias:

1. Cabeçalho: informações como nome completo, registro, leito e data devidamente preenchidos;

2. Conteúdo: existência de registro da presença de dor, bem como de sua medição, em evoluções, folha de monitorização clínica, admissão do paciente; profissional responsável pelo registro; registro das características da dor; analgesia prescrita; relatos dos profissionais sobre a satisfação do paciente com a analgesia e aplicação de medidas não farmacológicas para o alívio da dor.

Este estudo foi aprovado pelo Comitê de Ética em Pesquisa da instituição, obtendo parecer favorável no 063/2009.

\section{RESULTADOS}

A tabela 1 apresenta os dados referentes a característica, localização e intensidade da dor registrado nos prontuários.

\begin{tabular}{lcc}
\multicolumn{3}{l}{ Tabela 1 - Dados sobre dor registrados nos prontuários $(\mathrm{n}=63)}$. \\
\hline Variáveis & $\mathrm{n}$ & $(\%)$ \\
\hline Características da dor $(\mathrm{n}=57)$ & 57 & $(100,0)$ \\
Localização & 35 & $(61,4)$ \\
Fatores de melhora e piora & 25 & $(43,8)$ \\
Intensidade & 16 & $(28,0)$ \\
Frequência & 15 & $(26,3)$ \\
Qualidade & 10 & $(17,5)$ \\
Prejuízos advindos da dor & & \\
Localização da dor $(\mathrm{n}=57)$ & 30 & $(52,6)$ \\
Abdômen & 29 & $(50,9)$ \\
Cabeça & 19 & $(33,3)$ \\
Tórax & 17 & $(29,8)$ \\
Coluna lombar & 14 & $(24,6)$ \\
Membros inferiores & 10 & $(17,5)$ \\
Membros superiores & 8 & $(14,0)$ \\
Coluna cervical &
\end{tabular}

Tabela 1 - continuação

\begin{tabular}{lcc}
\hline Variáveis & $\mathrm{n}$ & $(\%)$ \\
\hline Outras* & 15 & $(26,3)$ \\
Intensidade da dor $(\mathrm{n}=25)$ & & \\
Intensa & 14 & $(56,0)$ \\
Leve & 7 & $(28,0)$ \\
Moderada & 4 & $(16,0)$ \\
\hline
\end{tabular}

*Hipogástrio (4); articulações (4); ombro (4); flancos (3); ânus (3); baixo ventre (3); boca (2); dorso (2); região inguinal (2); mesogástrio (1); cotovelo (1); joelho (1); esterno (1); orofaringe (1); pelve (1).

A tabela 2 apresenta os resultados da análise dos registros correspondentes à analgesia implementada, conforme prescrita e evoluída nos prontuários dos pacientes com SIDA internados.

Tabela 2 - Dados sobre analgesia registrados nos prontuários $(\mathrm{n}=63)$.

\begin{tabular}{lcc}
\hline Variáveis & $\mathrm{n}$ & $(\%)$ \\
\hline Analgésicos prescritos $(\mathrm{n}=63)$ & & \\
AINES* & 42 & $(66,7)$ \\
Analgésicos simples & 28 & $(44,4)$ \\
Adjuvantes & 26 & $(41,3)$ \\
Opioides & 19 & $(30,2)$ \\
Medida não farmacológica prescrita $(\mathrm{n}=7)$ & & \\
Aplicação de calor/frio & 3 & $(42,8)$ \\
Solução tópica & 2 & $(28,6)$ \\
Caminhada & 1 & $(14,3)$ \\
Relaxamento & 1 & $(14,3)$ \\
\hline *antinflamtórios ño
\end{tabular}

*anti-inflamatórios não esteroides.

A tabela 3 apresenta os dados referentes a qualidade da dor, bem como os fatores de alívio/agravo e os prejuízos gerados pela dor para o paciente.

Tabela 3 - Qualidade, fatores de melhora/piora e prejuízos gerados pela dor registrados nos prontuários $(n=60)$.

\begin{tabular}{lcc}
\hline Variáveis & $\mathrm{n}$ & $(\%)$ \\
\hline Qualidade $(\mathrm{n}=15)$ & & \\
Que irradia & 5 & $(33,3)$ \\
Pulsátil & 3 & $(20,0)$ \\
Em queimação & 1 & $(6,7)$ \\
Em pontada & 1 & $(6,7)$ \\
Em aperto & 1 & $(6,7)$ \\
Alodínia & 1 & $(6,7)$
\end{tabular}


Tabela 3 - continuação

\begin{tabular}{lll}
\hline Variáveis & $\mathrm{n}$ & $(\%)$ \\
\hline Tipo cólica & 1 & $(6,7)$ \\
Generalizada & 1 & $(6,7)$ \\
Fatores de melhora $(\mathrm{n}=10)$ & & \\
$\quad$ Medicações & 6 & $(60,0)$ \\
Repouso & 2 & $(20,0)$ \\
Alimentação & 1 & $(10,0)$ \\
Evacuação & 1 & $(10,0)$ \\
Fatores de piora $(\mathrm{n}=25)$ & & \\
Palpação & 5 & $(20,0)$ \\
Eliminações & 5 & $(20,0)$ \\
Mobilização & 4 & $(16,0)$ \\
Respiração (inspiração) & 4 & $(16,0)$ \\
Alimentação & 3 & $(12,0)$ \\
Deambulação & 1 & $(4,0)$ \\
Tosse & 1 & $(4,0)$ \\
Deglutição & 1 & $(4,0)$ \\
Atividades de vida diária & 1 & $(4,0)$ \\
\hline
\end{tabular}

\section{DISCUSSÃO}

Anotações sobre dor foram encontradas na maioria dos prontuários, porém para completa avaliação da dor, devem ser registradas as informações colhidas, as estratégias utilizadas para o seu controle, permitindo compartilhar os dados entre os diversos profissionais, melhorando a assistência ${ }^{7}$, e não apenas a descrição do local e sua intensidade ${ }^{8}$. As localizações coincidem com as citadas em outras pesquisas com o mesmo tipo de população ${ }^{3,9}$. Pesquisa ${ }^{9}$ envolvendo 103 adultos com SIDA em estágio avançado evidenciou dor mais prevalente nos membros inferiores (66\%) seguida da boca $(50,5 \%)$, cabeça $(42,3 \%)$, orofaringe $(39,8 \%)$ e tórax $(17,5 \%)$, localizações similares as do presente estudo.

A intensidade da dor foi muito semelhante as do estudo ${ }^{8}$ sobre dor e analgesia em pacientes internados, no qual a intensidade da dor avaliada pelos pacientes coincidiu com os registros dos profissionais.

Recente estudo ${ }^{10}$ com 302 pacientes ambulatoriais encontrou diferenças na intensidade da dor, mensurada com escala numérica e mostrou que a maioria dos pacientes $(53 \%)$ referiu dor leve, $20 \%$ dor moderada e $27 \%$ dor intensa. Os resultados comprovam o aumento da intensidade da dor com a progressão da doença, pois quanto mais a doença avança, maior é a incidência e intensidade da dor. Além disso, a intensidade da dor varia dependendo dos cuidados e do tratamento ${ }^{1}$.
A intensidade da dor, documentada na maior parte dos prontuários da presente pesquisa, não foi baseada na utilização de instrumentos padronizados para medição da dor, e sim nas análises individuais dos profissionais, mostrando a necessidade da avaliação sistemática da dor na instituição.

Não obstante a avaliação da dor apresentar componente subjetivo; devem ser usados instrumentos para uniformizar o acompanhamento dos pacientes com dor, como escalas uni e multidimensionais, questionários e índices, que além de quantificar a intensidade da dor, avaliam seu impacto no cotidiano e qualidade de vida do paciente ${ }^{11}$. Estudo $^{12}$ que aferiu a documentação da enfermagem relativa à avaliação da dor no pós-operatório evidenciou que a avaliação foi baseada, principalmente, no autorrelato dos pacientes, e que menos de $10 \%$ dos prontuários continha anotações sobre o uso sistemático de um instrumento de medição da dor, e que a localização da dor foi documentada em $50 \%$ dos prontuários e a característica da dor em apenas $12 \%$, dados que diferem dos encontrados neste estudo.

Quanto à duração da dor, os dados da presente pesquisa corroboram os resultados de outros estudos, ressaltando a persistência e o aumento da intensidade da dor conforme a doença progride ${ }^{1-3,9-10}$.

Em relação ao tratamento não farmacológico, na grande maioria dos prontuários, não havia quaisquer registros de sua utilização, no entanto existem pesquisas demonstrando o benefício dessas terapias no manuseio do paciente com dor, como mostra ensaio clínico randomizado com 79 pacientes, que comprovou o benefício potencial da arte terapia para redução dos sintomas associados à SIDA, incluindo a dor ${ }^{13}$.

Em recente estudo retrospectivo foram analisados registros de dor nos prontuários de crianças submetidas a cirurgias, e apenas $11,9 \%$ dos registros relacionavam prescrição de estratégias não farmacológicas para o alívio da dor, evidenciando pequena utilização deste método ${ }^{7}$.

O aumento na prescrição de adjuvantes analgésicos evidenciado neste estudo pode estar relacionado ao fato de que, aproximadamente $40 \%$ da dor na SIDA têm características neuropáticas ${ }^{1}$.

Quanto à satisfação com a analgesia prescrita, neste estudo houve registros que o paciente havia relatado melhora importante do quadro álgico, sem especificar o grau de melhora e as repercussões na evolução.

É evidente a importância da avaliação da dor como o $5^{\circ}$ sinal vital nas instituições de saúde em geral ${ }^{14}$, o que implica em avaliar a qualidade da dor e os fatores associados à sua melhora ou piora, para um cuidado mais direcionado à eficácia analgésica. $\mathrm{O}$ enfermeiro é fundamental para o controle da dor na avaliação, na interven- 
ção e na monitorização do tratamento, o que é facilitado se houver boa comunicação com o paciente ${ }^{15}$.

A existência de queixa dolorosa deve ser investigada durante toda a internação, incluindo a caracterização do local, a intensidade, a frequência, a duração e a qualidade do sintoma e deve ser registrada em instrumentos desenvolvidos pela instituição ${ }^{8}$.

$\mathrm{O}$ conhecimento da qualidade e dos fatores de melhora e de piora da dor (Tabela 3) é indispensável para o estabelecimento de metas para o cuidado do paciente com SIDA, pois norteia as ações do enfermeiro e do médico na prescrição das melhores alternativas analgésicas. Naqueles pacientes em que a mobilização e a deambulação agravam a dor, os profissionais devem promover o repouso e proporcionar mais conforto sem descuidar de medidas protetivas como a mudança de decúbito e os cuidados básicos de higiene, que embora possam causar dor, são imprescindíveis, e devem ser antecedidos pela aplicação de um analgésico de resgate, além dos fármacos prescritos para serem aplicados em horários pré-determinados.

A inexistência de instrumentos de avaliação padronizados na instituição não impediu que a dor fosse avaliada e registrados dados relativos ao início da queixa, localização, intensidade, qualidade, frequência e duração, fatores de melhora e piora. Entretanto, a adoção de um padrão de avaliação álgico diário do paciente pode contribuir para o aperfeiçoamento da assistência. É preciso que a equipe de saúde conheça a sua responsabilidade frente ao paciente com dor, sendo necessárias técnicas sistematizadas de avaliação para construir diagnósticos e identificar as intervenções adequadas para o alívio humanizado da dor ${ }^{14}$.

Nos prontuários estudados, a caracterização da dor quanto à qualidade e a descrição dos fatores de melhora/piora superaram os valores encontrados na literatura, quanto aos registros em prontuários de pacientes internados.

Os prontuários mostraram características relevantes sobre dor em pacientes com SIDA, com destaque para as anotações sobre presença, localização, duração e intensidade da dor na maioria dos pacientes. Entretanto, como a instituição não utiliza instrumento padronizado para esses registros é mais difícil prestar assistência qualificada, o que pode ser mais bem equacionado com a adoção de instrumentos adequados para a avaliação e registro dos dados relativos à dor.

O registro relativo ao tratamento não farmacológico pode facilitar a utilização desses recursos terapêuticos que somados aos farmacológicos, poderão colaborar de modo relevante para melhorar a qualidade de vida dos pacientes com dor.

\section{CONCLUSÃO}

É necessária a atenção dos profissionais para o registro de informações detalhadas das queixas álgicas dos pacientes com SIDA, com a adoção de instrumentos adequados para a avaliação e registro dos dados avaliados, para melhorar a assistência e o controle da dor que incide na maioria desses pacientes.

\section{REFERÊNCIAS}

1. Krashin DL, Merrill JO, Trescot AM. Opioids in the management of HIV-related pain. Pain Physician. 2012;15(3 Suppl):S157-68.

2. Johnson A, Condon KD, Mapas-Dimaya AC, et al. Report of an HIV clinic-based pain management program and utilization of health status and health service by HIV patients. J Opioid Manag. 2012;8(1):17-27.

3. Nair SN, Mary TR, Prarthana S, et al. Prevalence of pain in patients with HIV/AIDS: a cross-sectional survey in South Indian state. J Palliative Care. 2009;15(1):67-70. 4. Bottega FH, Fontana RT. A dor como quinto sinal vital: utilização da escala de avaliação por enfermeiros de um hospital geral. Texto Contexto Enferm. 2010;19(2):283-90. 5. Silva MS, Pinto, MA, Gomes LMX, et al. Dor na criança internada: a percepção da equipe de enfermagem. Rev Dor. 2011;12(4):314-20.

6. Campos JF, Souza SROS, Saurusaitis AD. Auditoria de prontuário: avaliação dos registros de aspiração traqueal em terapia intensiva. Rev Eletr Enf. 2008;10(2):358-66. 7. Tacla MTGM, Hayashida M, Lima RAG. Registro de dor pós-operatória em crianças: uma análise retrospectiva de hospitais de Londrina, PR, Brasil. Rev Bras de Enferm. 2008;61(3):289-95.

8. Silva YB, Pimenta CAM. Análise dos registros de enfermagem sobre dor e analgesia em doentes hospitalizados. Rev Esc Enferm USP. 2003;37(2):109-18.

9. Norval DA. Symptoms and sites of pain experienced by AIDS patients. S Afr Med J. 2004;94(6):450-4.

10. Namisango E, Harding R, Atuhaire L, et al. Pain among ambulatory HIV/AIDS patients: multicenter study of prevalence, intensity, associated factors, and effect. J Pain. 2012;13(7):704-13.

11. Martinez JE, Grassi DC, Marques LG. Análise da aplicabilidade de três instrumentos de avaliação de dor em distintas unidades de atendimento: ambulatório, enfermaria e urgência. Rev Bras Reumatol. 2011;51(4):299-308.

12. Idvall E, Ehrenberg A. Nursing documentation of postoperative pain management. J Clin Nurs. 2002;11(6):734-42. 
13. Rao D, Nainis N, Williams L, et al. Art therapy for relief of symptoms associated with HIV/AIDS. AIDS Care. 2009;21(1):64-9.

14. Saça CS, Carmo FA, Arbuleia JPS, et al. A dor como $5^{\circ}$ sinal vital: atuação da equipe de enfermagem no hospital privado com gestão do Sistema Único de Saúde
(SUS). J Health Sci Inst. 2010;28(1):35-41.

15. Costa AIS, Chaves MD. Dor em pacientes oncológicos sob tratamento quimioterápico. Rev Dor. 2012;13(1):45-9.

Apresentado em 10 de setembro de 2012.

Aceito para publicação em 12 de novembro de 2012. 